\title{
Massive Electrodynamic Gravity
}

\author{
Lukasz Andrzej Glinka* \\ B.M. Birla Science Centre, Hyderabad, India \\ *Corresponding author: laglinka@gmail.com; lukaszglinka@wp.eu
}

Received May 24, 2014; Revised June 02, 2014; Accepted June 09, 2014

\begin{abstract}
In this paper, an effcient combination of the diverse theoretical approaches, such like the Einstein gravitational waves, the Lifshitz cosmological perturbation theory, the Veltman perturbative quantum gravity, and the Maxwell electrodynamics, leads to an essentially new discussion of either massless and massive gravitons. The new force law of gravitation is established. The case of the Planckian particle is considered in the context of the Markov hypothesis.
\end{abstract}

Keywords: graviton mass, Maxwell electrodynamics, perturbative quantum gravity, gauge field theories, cosmological perturbation the- ory, gravitational waves, massless graviton, massive graviton, Planck- ian particle, Markov hypothesis, law of gravitation

Cite This Article: Lukasz Andrzej Glinka, "Massive Electrodynamic Gravity." Applied Mathematics and Physics, vol. 2, no. 3 (2014): 112-118. doi: 10.12691/amp-2-3-7.

\section{Introduction}

Existence of gravitational radiation is one of the proposals due to General Relativity, for the first time considered through Albert Einstein about one hundred years ago [1,2]. Let us present the core basis of this idea, the detailed derivations can be found in handbooks, Cf. the Refs. [3,4,5].

Gravitational waves are defined through the weak field condition

$$
\begin{aligned}
& g_{\mu v}=\eta_{\mu v}+h_{\mu v}, g^{\mu v}=\eta^{\mu v}-h^{\mu v}, \\
& h^{\mu v}=\eta^{\mu \alpha} \eta^{\mu \beta} h_{\alpha \beta}, \operatorname{det} h_{\mu v} \ll 1,
\end{aligned}
$$

where $\eta_{\mu v}=\operatorname{diag}(1,1,1,1)$ is the Minkowski metric in the relativistic convention. Assuming the coordinate transformation $x_{\mu}^{\prime}=\Lambda_{\mu}^{v} x_{v}$, where $\Lambda_{\mu}^{v}$ are the Lorentz matrices, the Lorentz invariance

$$
g_{\mu v}=\Lambda_{\mu}^{\alpha} \Lambda_{\mu}^{\beta} g_{\alpha \beta}, \eta_{\mu v}=\Lambda_{\mu}^{\alpha} \Lambda_{\mu}^{\beta} \eta_{\alpha \beta},
$$

leads to

$$
g_{\alpha \beta}=\eta_{\alpha \beta}+h_{\alpha \beta}, h_{\alpha \beta}=\Lambda_{\alpha}^{\mu} \Lambda_{\beta}^{v} h_{\mu v}
$$

The change of coordinates

$$
x^{\alpha}=x^{\alpha}+\xi^{\alpha}\left(x^{\beta}\right),
$$

generates the Lorentz transformation $\alpha$

$$
\Lambda_{\beta}^{\alpha}=\frac{\partial x^{\prime \alpha}}{\partial x^{\beta}}=\delta_{\beta}^{\alpha}+\partial_{\beta} \delta^{\alpha},
$$

which leads to the gauge transformation for Einstein's gravitational waves $\alpha$

$$
g_{\alpha \beta}^{\prime}=\eta_{\alpha \beta}+h_{\alpha \beta}-2 \partial_{(\beta} \xi_{\alpha)}, \xi_{\beta}=\eta_{\alpha \beta} \xi^{\alpha} .
$$

In such an approximation

$$
\begin{gathered}
\Gamma_{\mu v}^{\alpha}=\frac{1}{2} \eta^{\alpha \beta}\left(\partial_{\mu} h_{v \beta}+\partial_{v} h_{\beta \mu}-\partial_{\beta} h_{\mu v}\right), \\
R_{\mu v \alpha \beta}=2 \eta_{\mu v} \partial_{[\alpha} \Gamma_{v \beta]}^{\lambda}=\partial_{\alpha} \partial_{[v} h_{\mu] \beta}+\partial_{\beta} \partial_{[\mu} h_{v] \alpha}, \\
R_{\mu v}=\eta^{\alpha \beta} R_{\mu v \alpha \beta}=\partial_{\alpha} \partial_{(v} h_{\mu)}^{\alpha}-\frac{1}{2}\left(\partial_{\mu} \partial_{v} h+\square h_{\mu v}\right), \\
R=\eta^{\mu v} R_{\mu v}=\partial_{\alpha} \partial^{\mu} h_{\mu}^{\alpha}-\square h, \\
G_{\mu v}=\partial_{\alpha} \partial_{(v} h_{\mu)}^{\alpha}-\frac{1}{2}\left(\partial_{\mu} \partial_{v} h+\square h_{\mu v}\right. \\
\left.+\eta_{\mu v} \partial_{\alpha} \partial_{\beta} h^{\alpha \beta}+\eta_{\mu v} \square h\right),
\end{gathered}
$$

where $h=\eta^{\mu v} h_{\mu v}=h_{\mu}^{\mu}$ and $\square=\eta^{\mu v} \partial_{\mu} \partial_{v}$, and the Einstein field equations for gravitational waves are

$$
\begin{aligned}
& \partial_{\mu} \partial_{v} h+\square h_{\mu v}+\eta_{\mu v} \partial_{\alpha} \partial_{\beta} h^{\alpha \beta}+\eta_{\mu v} \square h \\
& -2 \partial_{\alpha} \partial_{(v} h_{\mu)}^{\alpha}=-2 \kappa T_{\mu v},
\end{aligned}
$$

where $T_{\mu v}$ is the energy-momentum tensor of Matter fields. Making use of the tensor

$$
\bar{h}_{\mu v}=h_{\mu v}-\frac{1}{2} \eta_{\mu v} h,
$$

and require the Lorenz-like gauge

$$
\partial^{\mu} \bar{h}_{\mu v}=0,
$$

the field equations (12) become the wave equation

$$
\square \bar{h}_{\mu v}=-2 \kappa T_{\mu v},
$$


whose 00-component and the spherical symmetry

$$
\bar{h}_{00}=\bar{h}_{00}(r),
$$

for the energy-momentum tensor with

$$
T_{00}=\rho c^{2},
$$

gives the Poisson equation

$$
\Delta \phi=\frac{4 \pi G}{c^{2}} \rho,
$$

with the gravitational potential

$$
\phi=-\frac{1}{4} \bar{h}_{00},
$$

which establishes the Newton law of universal gravitation. Then, neglecting other components of $\bar{h}_{\mu \nu}$,

$$
h=\bar{h}_{00}
$$

one has

$$
h^{00}=h^{11}=h^{22}=h^{33}=-2 \phi,
$$

and, consequently, the space-time is characterized by the interval

$$
d s^{2}=-(1+2 \varphi) d x^{0} d x_{0}+(1-2 \phi) d x^{i} d x_{i}
$$

Gravitational waves are one of the most intriguing questions of modern physics [6,7,8]. In 2011, V.F. Mukhanov presented the lecture on massive gravity [9] following from Einstein's gravitational waves, cosmological perturbation theory, and perturbative quantum gravity. This paper shows that the situation is really much more sophisticated than Mukhanov indicated.

\section{Cosmological Perturbations}

In the cosmological perturbation theory $[10,11]$

$$
\begin{aligned}
& d s^{2}=-(1+2 \Phi)\left(d x^{0}\right)^{2}+2 \partial_{i} B d x^{i} d x^{0} \\
& +\left[(1-2 \Psi) \delta_{i j}+2 s_{i j}\right] d x^{i} d x^{j},
\end{aligned}
$$

where the scalar and the tensor perturbations are

$$
\begin{gathered}
\Psi=-\frac{1}{6} h_{k}^{k}, \\
s_{i j}=\frac{1}{2}\left(h_{i j}+2 \Psi \delta_{i j}\right),
\end{gathered}
$$

while $\Phi$ is the gravitational potential responsible for the appropriate law of gravitation, and $\partial_{\mathrm{i}} \mathrm{B}$ is the vector perturbation. Then, the weak field is

$$
h_{00}=-2 \Phi, h_{0 i}=\partial_{\mathrm{i}} \mathrm{B}, \mathrm{h}_{i j}=2 s_{i j}-2 \Psi \delta_{i j},
$$

while the condition det $h_{\mu v} \ll 1$ gives

$$
\operatorname{det} h_{i j} \leq \frac{1+\left|\partial_{\mathrm{i}} \mathrm{B}\right|}{2|\Phi|} \text {. }
$$

However, this state of affairs differs from General Relativity, because the cosmological perturbation theory corresponds with fixation of an inertial reference frame in the background Minkowski space-time and the scalarvector- tensor decomposition of the perturbation metric components is made according to their transformation properties under spatial rotations, and is similar to decomposition of the electromagnetic field into electric and magnetic fields. The $(0,2)$ spatial tensor can be further decomposed into a trace and a trace- free part, which corresponds with looking for an irreducible representations of the rotation group.

For the metric (23), one has

$$
\begin{gathered}
\Gamma_{00}^{0}=\partial_{0} \Phi, \Gamma_{i 0}^{0}=\partial_{i} \Phi, \Gamma_{i j}^{0}=-\partial_{i} \partial_{j} B+\frac{1}{2} \partial_{0} h_{i j}, \\
\Gamma_{j 0}^{i}=\frac{1}{2} \partial_{0} h_{i j}, \Gamma_{00}^{i}=\partial^{i}\left(\Phi+\partial_{0} B\right), \\
\Gamma_{j k}^{i}=\partial_{(i} h_{k)}^{i}-\partial^{i} h_{j k},
\end{gathered}
$$

and

$R_{0 i 0 j}=\partial_{i} \partial_{j} \partial^{i}\left(\Phi+\partial_{0} B\right)-\frac{1}{2} \partial_{0}^{2} h_{i j}, R_{0 i j k}=-\partial_{0} \partial_{[j} h_{k] i}$,

$R_{i j k l}=2 \partial_{[j} \partial_{[k} h_{l] i]}$,

and

$$
\begin{gathered}
R_{00}=\Delta\left(\Phi+\partial_{0} B\right)+3 \partial_{0}^{2} \Psi \\
R_{0 i}=\partial_{0} \partial_{k}\left(3 \delta_{i}^{k} \Psi+\frac{1}{2} h_{i}^{k}\right) \\
R_{i j}=-\partial_{i} \partial_{j}\left(\Phi-3 \Psi+\partial_{0} B\right)-\frac{1}{2} \square h_{i j}+\partial_{k} \partial_{i} h_{j)}^{k} .
\end{gathered}
$$

For this reason

$$
R=6 \square \Psi-2 \Delta\left(\Phi+\partial_{0} B\right)+\partial_{i} \partial_{j} h^{i j},
$$

and, therefore,

$$
\begin{gathered}
G_{00}=3 \Delta \Psi+\frac{1}{2} \partial_{k} \partial_{l} h^{k l}, \\
G_{0 i}=\partial_{0} \partial_{k}\left(3 \delta_{i}^{k} \Psi+\frac{1}{2} h_{i}^{k}\right), \\
G_{i j}=\left(\delta_{i j} \Delta-\partial_{i} \partial_{j}\right)\left(\Phi+\partial_{0} B\right)-3\left(\delta_{i j} \square-\partial_{i} \partial_{j}\right) \Psi
\end{gathered}
$$$$
-\frac{1}{2} \square h_{i j}+\partial_{k} \partial_{(i} h_{j)}^{k}-\frac{1}{2} \delta_{i j} \partial_{k} \partial_{l} h^{k l} \text {. }
$$

Consequently, the Einstein field equations are

$$
\begin{gathered}
3 \Delta \Psi+\frac{1}{2} \partial_{k} \partial_{l} h^{k l}=\kappa T_{00}, \\
\partial_{0} \partial_{k}\left(3 \delta_{i}^{k} \Psi+\frac{1}{2} h_{i}^{k}\right)=\kappa T_{0 i}, \\
\left(\delta_{i j} \Delta-\partial_{i} \partial_{j}\right)\left(\Phi+\partial_{0} B\right)-3\left(\delta_{i j} \square-\partial_{i} \partial_{j}\right) \Psi \\
-\frac{1}{2} \square h_{i j}+\partial_{k} \partial_{(i} h_{j)}^{k}-\frac{1}{2} \delta_{i j} \partial_{k} \partial_{l} h^{k l}=\kappa T_{i j},
\end{gathered}
$$


and, amazingly, can be solved. The 00 component and $0 \mathrm{i}$ component give

$$
\partial_{0} T_{00}=\partial^{i} T_{0 i},
$$

while the $0_{\mathrm{j}}$-component leads to

$$
h_{j}^{k}=-6 \delta_{j}^{k} \Psi+2 \kappa \iint T_{0 j} d x^{0} d x^{k},
$$

Contraction of this solution with $\delta$ and application of (24) give

$$
\Psi=\frac{\kappa}{6} \iint T_{0 k} d x^{0} d x^{k},
$$

and, consequently, (42) becomes

$$
h_{j}^{i}=-\kappa \delta_{j}^{i} \iint T_{0 k} d x^{0} d x^{k}+2 \kappa \iint T_{0 j} d x^{0} d x^{i} .
$$

Noticing that

$$
\begin{gathered}
\partial_{k} \partial_{l} h^{k l}=\kappa T_{00}, \\
\partial_{k} \partial_{(i} h_{j)}^{k}=2 \kappa \int \partial_{(i} T_{0 j)} d x^{0}-\kappa \iint \partial_{i} \partial_{j} T_{0 k} d x^{0} d x^{k}, \\
\square h_{i j}=\kappa\left(\int \partial^{0} T_{0 k} d x^{k}-T_{00}\right) \delta_{i j} \\
+2 \kappa\left(\int \partial_{i} T_{0 i} d x^{0}-\int \partial^{0} T_{0 j} d x_{i}\right), \\
\square \Psi=\frac{\kappa}{6}\left(T_{00}-\int \partial^{0} T_{0 k} d x^{k}\right), \\
\partial_{i} \partial_{j} \Psi=\frac{\kappa}{6} \iint \partial_{i} \partial_{j} T_{0 k} d x^{0} d x^{k},
\end{gathered}
$$

one can see that the 00-component of the field equations becomes

$$
{ }_{\Delta} \Psi=\frac{\kappa}{6} T_{00}
$$

Making use of the relation (41), one receives

$$
\begin{gathered}
\int \partial^{0} T_{0 j} d x_{i}=\iint \partial_{0}^{2} T_{00} d x_{i} d x_{j}, \\
\int \partial^{0} T_{0 k} d x^{k}=\iint \partial_{0}^{2} T_{00} d x^{k} d x_{k}, \\
\iint \partial_{i} \partial_{j} T_{0 k} d x^{0} d x^{k}=\iint \partial_{i} \partial_{j} T_{00} d x^{k} d x_{k}, \\
\int \partial_{j} T_{0 i} d x^{0}=\int \partial_{j} T_{00} d x_{i},
\end{gathered}
$$

and, consequently, the ij component of the field equations is

$$
\begin{aligned}
& \left(\delta_{i j} \Delta-\partial_{i} \partial_{j}\right)\left(\Phi+\partial_{0} B\right) \\
& =\kappa T_{i j}+\frac{\kappa}{2}\left(T_{00}+\frac{2}{3} \iint \partial_{0}^{2} T_{00} d x^{k} d x_{k}\right) \delta_{i j} \\
& +\frac{\kappa}{2} \iint \partial_{i} \partial_{j} T_{00} d x^{k} d x_{k}-\kappa \iint \partial_{0}^{2} T_{00} d x_{i} d x_{j}-\kappa \int \partial_{j} T_{00} d x_{i},
\end{aligned}
$$

and after contraction with $\delta$ gives

$$
\Delta\left(\Phi+\partial_{0} B\right)=\frac{\kappa}{2}\left(T+T_{00}\right)
$$

where $T=\delta^{i j} T_{i j}$. Finally, one obtains

$$
\begin{gathered}
h_{i j}=2 \kappa \iint T_{00} d x_{i} d x_{j}-\kappa \delta_{i j} \iint T_{00} d x_{k} d x^{k}, \\
\Psi=\frac{\kappa}{6} \iint T_{00} d x_{k} d x^{k},
\end{gathered}
$$

and the condition (27) for non-zero T00 can be rewritten in the following form

$$
\frac{\kappa^{3}\left(\iint T_{00} d x_{k} d x^{k}\right)^{3}}{\operatorname{det}\left(\delta_{i j}-2 \frac{\iint T_{00} d x_{i} d x_{j}}{\iint T_{00} d x_{k} d x^{k}}\right)} \ll \frac{1+\left|\partial_{i} B\right|^{2}}{2|\Phi|} .
$$

In the equation (56), the evolutions of B and $\Phi$ can not be separated, but, consistency with the Newton gravitation suggests the Poisson equation

$$
{ }_{\Delta} \Phi=\frac{4 \pi G}{c^{2}} \rho,(60)
$$

and then

$$
{ }_{\Delta B}=\frac{\kappa}{2} \int T d x^{0},
$$

that is the problem is a choice of suitable $T_{\mu \nu}$.

\section{Perturbative Graviton}

Gauge field theories offer the perturbative point of view, wherein graviton is a spin-2 particle [12]. Then, $T_{\mu \nu}$ of graviton is derived from a Lagrangian

$$
L_{M}=L_{M}\left(\eta_{\mu v}\right)+\frac{1}{2} t^{\mu v}\left(h_{\mu v}-\eta_{\mu v}\right)+\ldots,
$$

or, explicitly

$$
T^{\mu v}=\left.2 \frac{\delta L_{M}}{\delta h_{\mu v}}\right|_{h^{\mu v}=\eta^{\mu v}}
$$

The Lagrangian of Matter fields has the form

$$
L_{M}=\frac{c^{6}}{\hbar^{2} G} \frac{m^{2}}{4}\left(h_{\mu}^{\mu} h_{v}^{v}-h_{\mu v} h^{\mu v}\right), h_{\mu}^{\mu}=h^{\mu v} h_{\mu v},
$$

where $\mathrm{m}$ is a graviton mass. Straightforward calculations gives

$$
T^{\mu v}=\frac{m^{2} c^{6}}{\hbar^{2} G} \eta^{\mu v},
$$

and, consequently,

$$
T=\delta^{i j} T_{i j}=3 \frac{m^{2} c^{6}}{\hbar^{2} G}, T_{00}=-\frac{m^{2} c^{6}}{\hbar^{2} G} .
$$

Implementing the massless limit, one has

$$
\Psi=0, h_{i j}=0, \Delta\left(\Phi+\partial_{0} B\right)=0,
$$

and, consequently, the metric (23) becomes

$$
d s^{2}=-(1+2 \Phi)\left(d x^{0}\right)^{2}+2 \partial_{i} B d x^{i} d x^{0}+d x^{i} d x^{j},
$$

while the condition (27) is equivalent to 


$$
\frac{1+\left|\partial_{i} B\right|^{2}}{2|\Phi|} \gg 0
$$

\section{Massless Limit}

The Laplace equation (67) can be rewritten in the form

$$
\Delta \Phi=-\Delta \partial_{0} B,
$$

and can be solved by the most general separation of variables $\Phi=\mathrm{C}, \partial_{0} \mathrm{~B}=\mathrm{C}$,

$$
\Delta \Phi=C, \Delta \partial_{0} B=-C,
$$

where $\mathrm{C}$ is a separation constant. In such a situation,

$$
\begin{aligned}
& \Phi(r)=C_{0}-\frac{C_{1}}{r}+\frac{C}{6} r^{2}, \\
& B(r, t)=\left(C_{0}^{\prime}-\frac{C_{1}^{\prime}}{r}-\frac{C}{6} r^{2}\right)\left(t-t_{0}\right) .
\end{aligned}
$$

Taking into account the boundary conditions

$$
\Phi\left(r_{0}\right)=\Phi_{0}, \partial_{i} \Phi\left(r_{0}\right)=\partial_{i} \Phi_{0},
$$

one receives

$$
\Phi(r)=\Phi_{0}+\left(1-\frac{r_{0}}{r}\right) r_{0}^{i} \partial_{i} \Phi_{0}+\frac{C}{6}\left(r^{2}-3 r_{0}^{2}+\frac{2 r_{0}^{3}}{r}\right)
$$

Because the constant in the gravitational potential

$$
\Phi_{0}-r_{0}^{i} \partial_{i} \Phi_{0}-\frac{C}{2} r_{0}^{2}
$$

vanishes when differentiation is performed, it does not play a role for the force law of gravitation $F_{i}=-m \partial_{i} \Phi$, and, consequently, it can be identically vanishing. This condition gives

$$
C=\frac{2}{r_{0}^{2}}\left(\Phi_{0}-r_{0}^{i} \partial_{i} \Phi_{0}\right)
$$

and, for this reason, one obtains finally i 2

$$
\Phi(r)=\frac{2}{3}\left(\Phi_{0}-\frac{5}{2} r_{0}^{i} \partial_{i} \Phi_{0}\right) \frac{r_{0}}{r}+\frac{\Phi_{0}-r_{0}^{i} \partial_{i} \Phi_{0}}{3} \frac{r^{2}}{r_{0}^{2}} .
$$

Considering

$$
\frac{2}{3}\left(\Phi_{0}-\frac{5}{2} r_{0}^{i} \partial_{i} \Phi_{0}\right)=-\frac{\Omega_{0}^{2}}{2} r_{0}^{2}, \frac{\Phi_{0}-r_{0}^{i} \partial_{i} \Phi_{0}}{3}=\frac{\omega_{0}^{2}}{2} r_{0}^{2}
$$

where $\Omega_{0}$ and $\omega_{0}$ are two constant angular frequency parameters, for which

$$
\Phi_{0}=\frac{1}{2}\left(5 \omega_{0}^{2}+\Omega_{0}^{2}\right) r_{0}^{2}, r_{0}^{i} \partial_{i} \Phi_{0}=\frac{1}{2}\left(2 \omega_{0}^{2}+\Omega_{0}^{2}\right) r_{0}^{2},
$$

and the force law of gravitation is 23

$$
F_{i}=\lim _{m \rightarrow \infty}\left(-\frac{m \Omega_{0}^{2}}{2} \frac{r_{0}^{3}}{r^{3}} r_{i}-m \omega_{0}^{2} r_{i}\right),
$$

one sees that the force law is non-trivial if and only if

$$
\Omega_{0}=\sqrt{\frac{K_{0}}{m}}, \omega_{0}=\sqrt{\frac{k_{0}}{m}},
$$

where $\mathrm{K} 0$ and $\mathrm{k} 0$ are positive constants.

\section{Massive Gravitons}

Let $\mathrm{m}$ be a positive graviton mass parameter. Then, one has

1. The Newton law of universal gravitation, if

$$
\omega_{0}=0, \Omega_{0}=\frac{G M}{r_{0}^{3}},
$$

where $\mathrm{M}>0$ is a mass of a spherically-symmetric body of radius $\mathrm{r} 0$

2. The oscillatory law of gravitation, if

$$
\omega_{0} \neq 0, \Omega_{0}=0 \text {. }
$$

3. The force law of gravitation $\sim \frac{1}{r^{2}}$, if

$$
r_{0}^{i} \partial_{i} \Phi_{0}=\Phi_{0}, \frac{2}{3}\left(\Phi_{0}-\frac{5}{2} r_{0}^{i} \partial_{i} \Phi_{0}\right)=\frac{M \Phi_{1}}{m},
$$

where $M$ is as previously, and $\Phi_{1}$ is a certain constant reference value of the potential $\Phi$. Then,

$$
\Phi_{0}=\frac{M}{m} \Phi_{1}, r_{0}^{i} \partial_{i} \Phi_{0}=-\frac{M}{m} \Phi_{1},
$$

and, for consistency, one has

$$
M=\beta m=\text { const, }
$$

where $\beta$ is a dimensionless constant, which means that the mass of a body generating gravitation is multiplication of the graviton mass.

Therefore,

$$
\Phi(r)=\beta \Phi_{1} \frac{r_{0}}{r}
$$

where the constant $\beta \Phi_{1} r_{0}$ can be established from experimental data.

4. The most general force law of gravitation, which is non-trivial in the massless limit,

$$
\Phi(r)=\frac{M_{1} \Phi_{1} r_{0}}{m} \frac{1}{r}+\frac{M_{2} \Phi_{2}}{m r_{0}^{2}} r^{2},
$$

where $M_{1} \Phi_{1}$ and $M_{2} \Phi_{2}$ are certain reference constants, is given by

$$
\Phi_{0}=\frac{5 M_{2} \Phi_{2}-M_{1} \Phi_{1}}{m}, r_{0}^{i} \partial_{i} \Phi_{0}=\frac{2 M_{2} \Phi_{2}-M_{1} \Phi_{1}}{m} .
$$

gives

$$
M_{2} \Phi_{2}=\frac{1}{2} M_{1} \Phi_{1}
$$

and, consequently,

$$
\Phi_{0}=\frac{3}{2} \frac{M_{1} \Phi_{1}}{m}, r_{0}^{i} \partial_{i} \Phi_{0}=0
$$


Therefore,

$$
\Phi(r)=\frac{2 \Phi_{0}}{3} \frac{r_{0}}{r}+\frac{\Phi_{0}}{3} \frac{r^{2}}{r_{0}^{2}},
$$

If $M_{1}=M$ and $\Phi_{1}=-\frac{G M}{r_{0}}$, then

$$
\Phi_{0}=-\frac{3}{2} \frac{G M^{2}}{m r_{0}},
$$

and, consequently,

$$
\Phi(r)=\frac{\omega_{0}^{2} r_{0}^{3}}{r}-\frac{\omega_{0}^{2}}{2} r^{2}, \omega_{0}=\sqrt{\frac{G M^{2}}{m r_{0}^{3}}}
$$

For $\mathrm{M}=\beta \mathrm{m}$ and $\mathrm{r}_{0}=\mathrm{R}$, where $\mathrm{R}$ if a radius of the spherical body,

$$
\omega_{0}=\sqrt{\beta} \Omega_{0}, \Omega_{0}=\frac{G M}{R^{3}} .
$$

Then, a graviton mass is

$$
m=\left(\frac{\Omega_{0}}{\omega_{0}}\right)^{2} M
$$

and suggests existence of the various gravitons emitted by a mass.

\section{Electromagnetic Gravity}

The resulting force law of gravitation

$$
F_{i}=-\frac{m \Omega_{0}^{2}}{2} \frac{r_{0}^{3}}{r^{3}} r_{i}-m \omega_{0}^{2} r_{i} .
$$

can be considered as the analogue of the Lorentz force

$$
F_{i}=m g_{i}+m \epsilon_{i}^{j k} v_{j} d_{k},
$$

where the field $d_{k}$ plays a role analogous to magnetic field, gravitational acceleration $g_{i}$ plays a role of electric field, and mass $\mathrm{m}$ of a moving particle plays a role of electric charge.

Introducing the analogue of the Faraday tensor of electromagnetic field

$$
G_{\mu v}=\frac{1}{\sqrt{4 \pi \epsilon_{0} G}}\left[\begin{array}{cc}
0 & \frac{g i}{c} \\
-\frac{g_{i}}{c} & \epsilon_{i j}^{k} d_{k}
\end{array}\right]
$$

for which the dual tensor is

$$
\widetilde{G}_{\mu \nu}=\epsilon_{\mu \nu k \lambda} \mathbb{G}^{\kappa \lambda}=\frac{1}{\sqrt{4 \pi \epsilon_{0} G}}\left[\begin{array}{cc}
0 & d_{i} \\
-d_{j} & -\epsilon_{i j}^{k} \frac{g_{k}}{c}
\end{array}\right]
$$

and taking the four-current

$$
J_{v}=\left|c \rho, J_{i}\right|,
$$

one obtains the analogue of the Maxwell equations

$$
\partial^{\mu} \mathbb{G}_{\mu v}=\mu_{0} J_{v}, \partial^{\mu} \widetilde{G}_{\mu v}=0,
$$

or, explicitly, the analogue of the Gauss law for electricity

$$
\partial^{i} g_{i}=-\sqrt{\frac{4 \pi G}{\epsilon_{0}}} \rho,
$$

the analogue of the Amp ere law

$$
\epsilon_{i j}^{k} \partial^{i} d_{k}=\sqrt{\frac{4 \pi G}{\epsilon_{0}}} \frac{1}{c^{2}} J_{i}-\frac{1}{c^{2}} \dot{g}_{i},
$$

the analogue of the Gauss law for magnetism

$$
\partial^{k} d_{k}=0,
$$

and the analogue of the Faraday law

$$
\dot{d}_{j}=\epsilon_{i j}^{k} \partial^{i} g_{k} .
$$

Introducing the analogue of the electromagnetic fourpotential $A_{\mu}$

$$
A_{\mu}=\frac{1}{\sqrt{4 \pi \epsilon_{0} G}} v_{\mu},
$$

where $v_{\mu}=\left[c, v_{i}\right]$ is the velocity four-vector, one has

$$
\mathbb{G}_{\mu v}=\partial_{\mu} A_{v}-\partial_{v} A_{\mu},
$$

and, making use of (108), one receives

$$
\begin{gathered}
G_{0 i}=-\frac{1}{\sqrt{4 \pi \epsilon_{0} G}} \frac{g_{i}}{c}=\partial_{0} A_{i}-\partial_{i} A_{0}=\frac{1}{\sqrt{4 \pi \epsilon_{0} G}} \frac{1}{c} \dot{v}_{i}, \\
G_{i j}=-\frac{1}{\sqrt{4 \pi \epsilon_{0} G}} \epsilon_{i j}^{k} d_{k}=\partial_{i} A_{j}-\partial_{j} A_{i} \\
=\frac{1}{\sqrt{4 \pi \epsilon_{0} G}}\left(\partial_{i} v_{j}-\partial_{j} v_{i}\right),
\end{gathered}
$$

or, equivalently,

$$
g_{i}=-\dot{v}_{i}, d_{i}=\epsilon_{i}^{k l} \partial_{k} v_{l} .
$$

Also, the stress-energy tensor is the analogue of the electromagnetic one

$T_{\mu v}=\frac{1}{\mu_{0}}\left(\eta^{\alpha \beta} \mathbb{G}_{\mu \alpha} \mathbb{G}_{\beta v}-\frac{1}{4} \eta^{\mu v} \mathbb{G}_{\alpha \beta} \mathbb{G}^{\alpha \beta}\right)=\left[\begin{array}{cc}\epsilon & \frac{S_{i}}{c} \\ \frac{S_{j}}{c} & -\sigma_{i j}\end{array}\right]($

where $\epsilon$ is the field energy density

$$
\epsilon=\frac{g^{2}+c^{2} d^{2}}{8 \pi G},
$$

$\mathrm{S}_{\mathrm{i}}$ is the analogue of the Poynting vector

$$
S_{i}=\frac{c}{4 \pi G} \epsilon_{i}^{k l} g_{k} d_{l}
$$

and $\sigma_{\mathrm{ij}}$ is the analogue of the Maxwell stress tensor

$$
\sigma_{i j}=\frac{1}{4 \pi G}\left(g_{i} g_{j}+c^{2} d_{i} d_{j}-\frac{g^{2}+c^{2} d^{2}}{2} \delta_{i j}\right) \text {. }
$$

Making use of the definitions (111) within the equation (104), one receives 


$$
\square v_{j}-\partial_{j}\left(\partial^{i} v_{i}\right)=\sqrt{\frac{4 \pi G}{\epsilon_{0}}} \frac{1}{c^{2}} J_{j},
$$

and, similarly, the equation (103) leads fo

$$
\partial^{i} \dot{v}_{i}=\sqrt{\frac{4 \pi G}{\epsilon_{0}}} \rho .
$$

Consequently,

$$
J_{\mu}=c^{2} \sqrt{\frac{\epsilon_{0}}{4 \pi G}}\left[\frac{1}{c} \partial^{i} \dot{v}_{i}, \square v_{j}-\partial_{j}\left(\partial^{i} v_{i}\right)\right] .
$$

Particularly, for a graviton mass $m=0$, one has

$$
J_{\mu}=-\frac{1}{\mu_{0}}\left(\frac{m c}{\hbar}\right)^{2} A_{\mu},
$$

or, taking into account (107),

$$
J_{\mu}=c^{2} \sqrt{\frac{\epsilon_{0}}{4 \pi G}}\left[-c\left(\frac{m c}{\hbar}\right)^{2},-\left(\frac{m c}{\hbar}\right)^{2} v_{j}\right] .
$$

Comparing the relations (118) and (120), one obtains

$$
\partial^{i} \dot{v}_{i}=-\left(\frac{m c^{2}}{\hbar}\right)^{2}, \square v_{j}-\partial_{j}\left(\partial^{i} v_{i}\right)=-\left(\frac{m c}{\hbar}\right)^{2} v_{j}
$$

and, consequently, one receives the analogue of the Proca equation

$$
\left(\square+\left(\frac{m c}{\hbar}\right)^{2}\right) v_{j}=0, m=\frac{\hbar}{c^{2}} \sqrt{\partial^{i} g_{i}},
$$

describing the massive graviton.

The analogy with the Maxwell electrodynamics gives the Lagrangian

$$
L=-\frac{1}{4 \mu_{0}} \mathbb{G}_{\mu v} \mathbb{G}^{\mu v}-A_{\mu} J^{\mu} .
$$

Also, on the quantum level, one obtains the analogue of the quantum electrodynamics and the Feynman diagrams are fully analogous. Making use of (119), the Lagrangian (123) becomes

$$
\begin{aligned}
L & =-\frac{c^{2}}{4 \pi G}\left[\frac{1}{2}\left(\partial_{\mu} v_{v} \partial^{\mu} v^{v}-\partial_{\mu} v_{v} \partial^{v} v^{\mu}\right)+\left(\frac{m c}{\hbar}\right)^{2} v_{\mu} v^{\mu}\right] \\
& =-\frac{c^{2}}{4 \pi G}\left[\frac{1}{2}\left(\partial_{\mu} v_{i} \partial^{\mu} v^{i}-\partial_{i} v_{j} \partial^{j} v^{i}\right)+\left(\frac{m c}{\hbar}\right)^{2} v_{i} v^{i}\right]
\end{aligned}
$$

where we have made use of the four-velocity definition $v_{\mu}$ $=\left[\mathrm{c}, \mathrm{v}_{\mathrm{i}}\right]$, and the constant Lagrangian has been omitted. Noticing that the continuity equation

$$
\partial^{\mu} J_{\mu}=0
$$

should be satisfied, the equation (119) gives the Lorentz gauge

$$
\partial^{\mu} A_{\mu}=0
$$

which gives the divergence-free velocity field

$$
\partial^{\mu} v_{\mu}=\partial^{i} v_{i}=0
$$

which implies that $\partial^{i} \dot{v}_{i}=0$ and, consequently, the graviton mass identically vanishes $\mathrm{m}=0$. However, because this is an additional relation which can be broken and which is not needed for consistency of the theory, one must not take this condition into account. When the continuity equation is non-valid, then a graviton mass is existent and fully argued.

The equations (97) and (98) can be used to find the explicit form of the fields $g_{i}$ and $d_{i}$. There are two identifications

$$
\begin{gathered}
g_{i}=-\frac{\Omega_{0}^{2}}{2} \frac{r_{0}^{3}}{r^{3}} r_{i}, \epsilon_{i}^{j k} v_{j} d_{k}=-\omega_{0}^{2} r_{i}, \\
g_{i}=-\omega_{0}^{2} r_{i}, \epsilon_{i}^{j k} v_{j} d_{k}=-\frac{\Omega_{0}^{2}}{2} \frac{r_{0}^{3}}{r^{3}} r_{i}
\end{gathered}
$$

For the first one

$$
\partial^{i} g_{i}=0
$$

and, consequently, $m=0$, and the Lorentz gauge is consistent. For the second one,

$$
\partial^{i} g_{i}=-3 \omega_{0}^{2}, m=\sqrt{3} \frac{\hbar}{c^{2}} \sqrt{-\omega_{0}^{2}},
$$

and for $\omega_{0}^{2}>0$ a graviton is a tachyon, whereas for $\omega_{0}^{2}=-\left|\omega_{0}^{2}\right|<0$, that is an inverted harmonic oscillator, one has

$$
m=\sqrt{3} \frac{\hbar\left|\omega_{0}\right|}{c^{2}},
$$

what compared with (96), gives

$$
M \Omega_{0}^{2}=-\sqrt{3} \frac{\hbar\left|\omega_{0}\right|^{3}}{c^{2}} .
$$

Therefore, for non-tachyonic massM, one should have $\Omega_{0}^{2}=-\frac{G M}{R^{3}}=-\left|\Omega_{0}\right|^{2}$, that is a repulsive gravitation, and

$$
\left|\omega_{0}\right|=\frac{1}{R}\left(\frac{G M^{2} c^{2}}{\sqrt{3} \hbar}\right)^{1 / 3} .
$$

Interestingly, for a sphere of radius equal to the Planck length $R=\ell_{P}$ and mass equal to the Planck mass $\mathrm{M}=\mathrm{M}_{\mathrm{P}}$, one obtains

$$
\begin{gathered}
\left|\omega_{0}\right|=\frac{\omega_{P}}{3^{1 / 6}} \approx 1.544 \cdot 10^{19} \mathrm{YHz}, \\
\left|\Omega_{0}\right|=\omega_{P} \approx 1.855 \cdot 10^{19} \mathrm{YHz}, \\
m=3^{1 / 3} M_{P} \approx 1.7610 \cdot 10^{4} \frac{\mathrm{YeV}}{c^{2}},
\end{gathered}
$$

where $\omega_{\mathrm{P}}$ is the Planck frequency, while the force law of gravitation becomes

$$
F_{i}=M_{P} \omega_{P}^{2}\left(1+\frac{3^{1 / 3}}{2} \frac{\ell_{P}^{3}}{r^{3}}\right) r_{i}
$$


For emission of a graviton through the Planckian particle, $\mathrm{M}=\mathrm{MP}$, a hy- pothetical particle existing in the Planck scale energetic regime, a graviton mass exceeds $\mathrm{MP}$, what in the light of the Markov hypothesis [13,14], that is $\mathrm{m} \leq \mathrm{MP}$, means that this graviton is undetectable.

Moreover, one can see that (111) gives

$$
\epsilon_{i}^{j k} v_{j} d_{k}=v^{l} \partial_{i} v_{l}-v_{l} \partial^{l} v_{i}
$$

and when the velocity field vi is known, for example as the solution to the equation (122), both (128) and (129) are the equations for $r_{i}$

$$
\begin{gathered}
r_{i}=R^{2}\left(\frac{G M^{2} c^{2}}{\sqrt{3} \hbar}\right)^{-2 / 3}\left(v^{j} \partial_{i} v_{j}-v_{j} \partial^{j} v_{i}\right), \\
\frac{r_{i}}{r^{3}}=\frac{2}{G M}\left(v^{l} \partial_{i} v_{l}-v_{l} \partial^{l} v_{i}\right) .
\end{gathered}
$$

\section{Summary}

Joining the various approaches to gravity - the Einstein gravitational waves following from General Relativity whose applicability is unclear, the Lifshitz cosmological perturbation theory which is cureently applicable for astrophysical scales, the Veltman perturbative quantum gravity based on the gauge field theories which usually are applicable for high energy physics, and the Maxwell electrodynamics which is applicable at any scales - we have received an essential discussion on either massless and massive gravitons. The analysis of the Planckian particle in the context of the Markov hypothesis has been shown that a massive graviton emitted throughout such a particle is undetectable. The new force law of gravitation has been established. This theory, however, needs a straightforward confrontation with a suitable experimental data. Its should be emphasized that the discussion of this model in more advanced detail was presented in the author's monograph [15].

\section{References}

[1] A. Einstein, Sitzungsber. Preuss. Akad. Wiss. 1, 688 (1916).

[2] A. Einstein, Sitzungsber. Preuss. Akad. Wiss. 1, 154 (1918).

[3] S. Weinberg, Gravitation and Cosmology. Principles and Applications of the General Theory of Relativity (John Wiley \& Sons, 1972).

[4] S. Carroll, Space-time and Geometry. An Introduction to General Rela- tivity (Addison-Wesley, 2004).

[5] L.D. Landau and E.M. Lifshitz, The Classical Theory of Fields. Course of Theoretical Physics, Volume 2 (ButterworthHeinemann, 1994).

[6] J.D.E. Craighton and W.G. Anderson, Gravitational-Wave Physics and Astronomy: An Introduction to Theory, Experiment and Data Analysis (Wiley-VCH, 2011).

[7] P. Jaranowski and A. Kr'olak, Analysis of Gravitational-Wave Data (Cambridge University Press, 2009).

[8] M. Maggiore, Gravitational Waves. Volume 1 Theory and Experiments (Oxford University Press, 2007).

[9] V.F. Mukhanov, Massive Gravity, talkat Quantum Theory and Gravita- tion, Eidgen“ossische Technische Hochschule, Zurich," Switzerland, June 14-24, 2011.

[10] E.M. Lifshitz, J. Phys. (USSR) 10, 116 (1946).

[11] E. Bertschinger, Cosmological Dynamics, in Cosmology and Large Scale Structure. Les Houches Summer School, Session LX, ed. by R.Schaeer, J. Silk, M. Spiro, and J. Zinn-Justin, (Elsevier, 1996), pp. 273-347.

[12] M.J.G. Veltman, Quantum Theory of Gravitation, in Methods in Field Theory. Les Houches, Session XXVIII, ed. by R. Balian and J. Zinn- Justin (North Holland, 1976), pp. 265-328.

[13] M.A. Markov, Prog. Theor. Phys. Suppl. E65, 85 (1965).

[14] M.A. Markov, Sov. Phys. JETP 24, 584 (1967).

[15] L.A. Glinka, AEthereal Multiverse: A New Unifying Theoretical Approach to Cosmology, Particle Physics, and Quantum Gravity (Cambridge In- ternational Science Publishing, 2012). 